\title{
A Randomized, Double-Blind, Placebo-Controlled Study of Preemptive Oral Oxycodone with Morphine Patient-Controlled Analgesia for Postoperative Pain Management in Patients Undergoing Uterine Artery Embolization for Symptomatic Uterine Fibroids
}

\author{
Alex H. Konstantatos • Helen Kavnoudias • \\ James R. Stegeman • Dana Boyd • Maryann Street • \\ Michael Bailey • Stuart M. Lyon • Kenneth R. Thomson
}

Received: 18 September 2013/ Accepted: 9 April 2014/Published online: 1 July 2014

(C) Springer Science+Business Media New York and the Cardiovascular and Interventional Radiological Society of Europe (CIRSE) 2014

\begin{abstract}
Purpose To evaluate the analgesic efficacy of oral premedication of oxycodone in a group of patients undergoing elective uterine artery embolization under sedation for fibroid disease.

Methods Thirty-nine patients (mean age 42.3 years) were prospectively randomized $1: 1$ to receive $20 \mathrm{mg}$ oxycodone or placebo orally immediately before their procedure. At the commencement of the procedure, patients were provided with a patient-controlled analgesia device for $24 \mathrm{~h}$, programmed to deliver $1 \mathrm{mg}$ boluses of intravenous morphine with a 5 min lockout. Mean visual analog scale pain
\end{abstract}

A. H. Konstantatos - D. Boyd

Department of Anaesthesia, The Alfred Hospital, Melbourne,

VIC 3004, Australia

e-mail: a.konstantatos@alfred.org.au

D. Boyd

e-mail: d.boyd@alfred.org.au

\section{A. H. Konstantatos}

Department of Medicine, Nursing and Health Sciences, Monash

University, Melbourne, VIC, Australia

H. Kavnoudias $(\bowtie) \cdot$ J. R. Stegeman · S. M. Lyon ·

K. R. Thomson

Department of Radiology, The Alfred Hospital, Melbourne,

VIC 3004, Australia

e-mail: h.kavnoudias@alfred.org.au

J. R. Stegeman

e-mail: jrstegeman@gmail.com

S. M. Lyon

e-mail: 1yonsey@optusnet.com.au

K. R. Thomson

e-mail: k.thomson@alfred.org.au intensity ratings $(0-100 \mathrm{~mm})$ were measured from both groups and evaluated over 0 to $6 \mathrm{~h}$ as the primary end point. Other measured parameters included opioid-related side effects and eligibility for discharge (NCT00163930; September 12, 2005).

Results Early pain intensity did not vary significantly between the active and placebo groups [mean (standard deviation): 3.2 (2.5) vs. 3.1 (2.2), $p=0.89$ ]. The oxycodone group, however, experienced significantly more nausea $(p=0.035)$ and a greater incidence of vomiting $(p=0.044)$. Overall opioid requirement over $24 \mathrm{~h}$, measured as oral morphine equivalent, was greater in the oxycodone group (median [interquartile range]: 64.5

M. Street

School of Nursing and Midwifery, Deakin University, Burwood,

VIC 3125, Australia

e-mail: maryann.street@deakin.edu.au

M. Bailey

Department of Epidemiology and Preventative Medicine,

Monash University, Melbourne, VIC, Australia

e-mail: michael.bailey@monash.edu

S. M. Lyon - K. R. Thomson

Department of Surgery, Monash University, Melbourne, VIC, Australia 
[45-90] mg vs. $22.5[15-46.5] \mathrm{mg}, p<0.0001)$. The number of patients first eligible for discharge at $24 \mathrm{~h}$ in the oxycodone group was decreased but not significantly $(p=0.07)$.

Conclusion The addition of preprocedural oral oxycodone to morphine patient-controlled analgesia does not offer any analgesic advantage to patients having uterine artery embolization and may cause a greater incidence of nausea and vomiting.

Keywords Uterine artery embolization · Opioids . Patient controlled analgesia . Pain management

\section{Introduction}

Transcatheter uterine artery embolization (UAE) was first described as a method to treat large symptomatic fibroids to avoid bleeding complications associated with open surgical myomectomy [1, 2]. Pain associated with UAE is described as severe and variable $[1,3]$ and it remains the primary reason for overnight admission. Pain is due to fibroid and transient myometrial ischemia and has been characterized through reference to records of patient-controlled analgesia (PCA) with opioid [4]. The natural pain history of UAE increases in intensity to a point approximately $2 \mathrm{~h}$ after the procedure, remaining at this level for several hours, then rapidly decreasing [4].

Preprocedural history of heavier menstrual bleeding, pelvic pain, pressure sensation, uterine and fibroid volume are not associated with intensity of postprocedure pain [5]. Procedural factors identified that may minimize pain after UAE include selection to avoid bilateral UAE among patients with limited fibroid disease [6], type of embolic material used, Contour SE requiring less opioid analgesia compared to Embosphere/Embosphere Gold [7, 8] and the use of preemptive analgesic, anti-inflammatory and antiemetic medications.

Numerous analgesic and systemic Non Steroidal AntiInflammatory Drug (NSAID) therapies have been described for the postprocedure pain management of UAE. NSAIDs have been described in randomized double-blind studies, with oral rofecoxib showing similar analgesic and opioid reducing effect to ibuprofen [9]. NSAID impregnated embolic beads have been shown to develop favorable concentrations in uterine tissues in animal models [10-12]. These provided superior analgesia compared to placebo beads (containing polyvinyl alcohol) after UAE in humans [13]. Morphine PCA has been evaluated in a number of randomized double-blind studies. Addition of ketamine to morphine PCA did not reduce morphine requirement in one study [14], while comparison to remifentanil target controlled infusion showed significant reduction of early Pain
Intensity (PI) in the remifentanil group [15]. Morphine PCA has also been compared to fentanyl PCA in a nonrandomized study, where it was found to produce lower PI scores [16].

Favorable descriptions of epidural analgesia for UAE exist only in case series without reference to control groups $[17,18]$, while intra-arterial lidocaine injection was associated with significantly lower pain scores but no reduction in morphine PCA requirements in a randomized doubleblind setting [19].

Sustained-release oral oxycodone is a strong analgesic that is simple and cheap to administer and that shows potential for treating pain in experimental human visceral pain models [20]. Early absorption of an oral dose of $20 \mathrm{mg}$ of sustained-release oxycodone is rapid, with significant increases in blood concentration seen by $40 \mathrm{~min}$, building to peak blood levels by $3.2 \mathrm{~h}$ [21], closely matching the profile of PI with UAE [4]. We undertook a prospective randomized double-blind study to evaluate the effects of a preprocedural oral dose of $20 \mathrm{mg}$ of oxycodone with UAE, our primary hypothesis being that oral preemptive analgesia with an opioid would lead to a reduction in early PI. Secondary hypotheses examined outcomes of $\mathrm{PI}$ at $24 \mathrm{~h}$, opioid-related side effects, satisfaction with pain management and readiness for discharge.

\section{Materials and Methods}

After approval from our intuition's Human Research and Ethics Committee (trial registration NCT00163930, September 12, 2005), a total of 42 women, mean age 42.3 years, American Society of Anesthesiologists (ASA) status I to III, gave written informed consent to participate before their scheduled elective UAE procedure. Participants had no allergy to oxycodone and morphine, no history of opioid analgesia requirement 2 weeks before the procedure and no preexisting hepatic or renal disease.

The study was randomized and double-blind; reporting was in accordance with CONSORT guidelines (Fig. 1). Patients were randomly assigned to receive $20 \mathrm{mg}$ of oral sustained-release oxycodone (OxyContin SR) or matched placebo by blinded researchers, according to a computergenerated simple randomization code. Group allocation was concealed and controlled by our institution's pharmacy department, thus ensuring blinding of patients, researchers and proceduralists. Other preprocedural medications provided to all patients were oral paracetamol $1.5 \mathrm{~g}$, pyroxicam $20 \mathrm{mg}$ and ondansetron $8 \mathrm{mg}$.

Procedures were performed or supervised by two interventional radiologists, both with over two decades of UAE experience. All procedures were bilateral, with local anesthetic infiltration of the access site and involved 
Fig. 1 CONSORT flow diagram. All patients who were randomly assigned to analgesia premedication were included in the intent-to-treat population

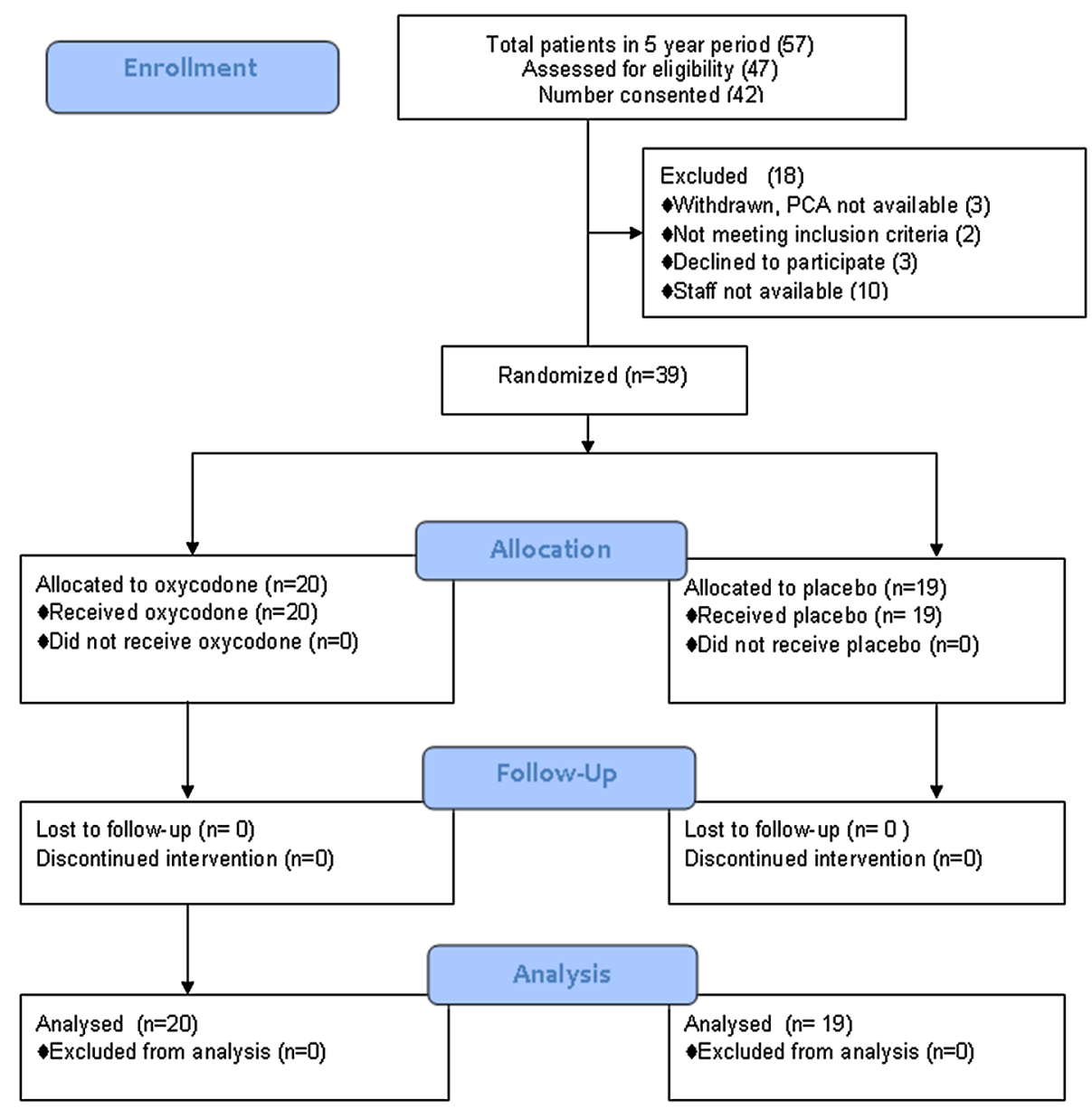

uterine artery injection of polyvinyl alcohol foam particles (500-700 um Contour SE; Cook Australia). End point was stasis of the main uterine artery confirmed for a minimum of five heartbeats. All participants had intravenous conscious sedation with midazolam and received antibiotics (Cephalothin $1 \mathrm{~g}$ ) and an antiemetic (Maxolon $10 \mathrm{mg}$ ). They were provided with a device for administering PCA programmed to deliver $1 \mathrm{mg}$ bolus morphine with a $5 \mathrm{~min}$ lockout interval (Gemstar; Abbot Laboratories, North Chicago, IL) up to $24 \mathrm{~h}$ after commencement of the procedure. Instructions were for participants to deliver morphine boluses as needed to control their pain. After PCA, patients were offered $5 \mathrm{mg}$ of oral oxycodone, immediate release (Endone), every $2 \mathrm{~h}$ if required.

Participants received supplemental oxygen throughout the procedure. They were monitored with continuous pulse oximetry, electrocardiography and noninvasive blood pressure measurements at $5 \mathrm{~min}$ intervals (Phillips Suresigns Medical Systems, Koninklijke-Philips Electronics, Andover, MA). Participants experiencing nausea/vomiting were immediately treated with $4 \mathrm{mg}$ intravenous ondansetron.

Demographic information included age, weight, coexisting health status, time from premedication to procedure commencement, procedure duration and name of interventionalist. Baseline size of uterus and number and size of fibroids were recorded.

PI was measured on a $10 \mathrm{~cm}$ visual analog scale (VAS) (left-hand margin representing no pain, right-hand margin representing the worst pain possible). PI was measured at 2, 4 and $6 \mathrm{~h}$ after commencement of the UAE and a mean level was calculated for the primary end point. PI was also measured at $24 \mathrm{~h}$ via VAS. Cumulative opioid requirement was measured with reference to the Abbot Gemstar. Opioid requirement was measured as intravenous morphine equivalent from the time of premedication until the patient ceased to require postprocedural opioid treatment after discharge, with $1 \mathrm{mg}$ of oral oxycodone taken to be equivalent to $0.5 \mathrm{mg}$ of intravenous morphine [22]. Patients were followed up by phone after discharge until they did not require oral opioid treatment.

Participants were encouraged to report any opioid-related side effects at any time during or after the procedure. Researchers enquired about nausea, vomiting and pruritus and measured the level of sedation hourly for the first $6 \mathrm{~h}$ and then every $4 \mathrm{~h}$ until discharge. Pruritus, nausea, vomiting and sedation were measured as secondary end points. 
Patients were deemed suitable for discharge if, on the next day, they had pain easily controlled by oral analgesics, nausea and/or vomiting easily controlled without the need for intravenous antiemetics and no uterine bleeding. Time to suitability for discharge was recorded as a secondary end point.

Satisfaction with analgesia was assessed using a $10 \mathrm{~cm}$ VAS (left-hand margin reflecting no satisfaction at all and right-hand margin reflecting fully satisfied). Participants also provided an assessment of their satisfaction with the whole experience (procedure, sedation and analgesia) using a 4 point scale where 1 reflected that they were unsatisfied, 2 a little, 3 moderately and 4 fully satisfied.

Mean Pain Intensity (MPI) data from a population of patients undergoing UAE at our institution formed the basis for power analysis. Assuming an alpha error of 0.05, a beta error of 0.2 , and a predicted difference of MPI score of $30 \%$, we aimed to recruit 40 patients.

MPI data for 0 to $6 \mathrm{~h}$, age, time from premedication to procedure commencement, procedure duration, and satisfaction of the procedure were tested for normality and analyzed by Student's $t$-test. Number of fibroids, mass of dominant fibroid, size of uterus, number of ampoules of embolic agent, PI at $24 \mathrm{~h}$, presence of nausea, opioid requirement, satisfaction and sedation scores showed nonparametric characteristics and were analyzed by Wilcoxon rank sum test. Binomial data, pruritus, respiratory depression, eligibility for discharge and interventionalist were analyzed by the chi-square or Fisher's exact test, as appropriate. $p$ values of less than 0.05 were considered significant.

\section{Results}

Recruitment was from November 2006 to March 2012. Fiftyseven UAE procedures were performed at our institution during this period. Forty-two eligible patients provided informed consent and 39 of 42 enrolled; 20 received preprocedural oral oxycodone and 19 placebo (Fig. 1). Three of 42 were withdrawn from the study because the PCA on the procedure day was not available, 2 of 57 were unsuitable because of comorbidities/disabilities, 3 of 57 declined and 10 of 57 were not queried about consent because trial interventionalists and/or research staff were not available.

Baseline characteristics between the two groups were similar for mean age, ASA ranking, size of uterus, number of fibroids, mass of dominant fibroid, volume and size of embolic agent, body mass index, time from premedication to procedure commencement, procedure duration and interventionalist (Table 1).

Technical success was $97.4 \%$ (38 of 39), with one patient going on to a total hysterectomy at 6 months. MPI
Table 1 Demographic characteristics of patients presenting for uterine embolization

\begin{tabular}{|c|c|c|c|}
\hline Characteristic & $\begin{array}{l}\text { Preoperative } \\
\text { placebo } \\
(n=19)\end{array}$ & $\begin{array}{l}\text { Preoperative } \\
\text { opioid } \\
(n=20)\end{array}$ & $p$ \\
\hline Age, y & $41.7(6.3)$ & $42.8(6.3)$ & 0.58 \\
\hline \multicolumn{4}{|l|}{ ASA class } \\
\hline I & $84 \%$ & $74 \%$ & \\
\hline II & $11 \%$ & $16 \%$ & \\
\hline III & $5 \%$ & $10 \%$ & \\
\hline Body mass index, $\mathrm{kg} / \mathrm{m}^{2}$ & $28.2(8.0)$ & $26.0(6.5)$ & 0.54 \\
\hline Size of uterus, $\mathrm{cm}^{3}$ & $\begin{array}{l}374 \\
\quad[249.5-969.0]\end{array}$ & $\begin{array}{l}512 \\
\quad[255.3-1200]\end{array}$ & 0.83 \\
\hline No. of fibroids & $1[1-2]$ & $1[1-3]$ & 0.34 \\
\hline Dominant fibroid, $\mathrm{cm}^{3}$ & $\begin{array}{l}538 \\
\quad[185.9-1950]\end{array}$ & $\begin{array}{l}512 \\
\quad[124-605.6]\end{array}$ & 0.43 \\
\hline $\begin{array}{l}\text { Premedication to start of } \\
\text { procedure time, min }\end{array}$ & $19.0(11.1)$ & $18.4(12.8)$ & 0.87 \\
\hline \multicolumn{4}{|l|}{ Embolic agent } \\
\hline No. of ampoules & $1[1-2]$ & $2[1-3]$ & 0.27 \\
\hline Particle size, $\mu \mathrm{m}$ & $600(70.71)$ & $596(88.38)$ & 0.89 \\
\hline Procedure duration, min & $74.7(30.0)$ & $66.9(22.2)$ & 0.49 \\
\hline \multicolumn{4}{|l|}{ Operator } \\
\hline 1 & $48 \%$ & $47 \%$ & 0.9 \\
\hline 2 & $52 \%$ & $53 \%$ & 1.0 \\
\hline
\end{tabular}

Data are presented as mean (standard deviation), median [interquartile range] or proportions as appropriate

ASA American Society of Anesthesiologists

ASA physical status classification system: (1) a normal healthy patient; (2) a patient with mild systemic disease; (3) a patient with severe systemic disease; (4) a patient with severe systemic disease that is a constant threat to life; (5) a moribundd patient who is not expected to survive without the operation; (6) a declared brain-dead patient whose organs are being removed for donor purposes

Table 2 Pain intensity ratings measured by visual analog scale at 0 to $6 \mathrm{~h}$ and $24 \mathrm{~h}$ after commencement of uterine embolization procedure

\begin{tabular}{llll}
\hline Pain intensity & Preoperative placebo & Preoperative opioid & $p$ \\
\hline $0-6 \mathrm{~h}$ & $3.1(2.2)$ & $3.2(2.5)$ & 0.89 \\
$24 \mathrm{~h}$ & $1.5[0.2-4.7]$ & $1.1[0.8-2.7]$ & 0.98 \\
\hline
\end{tabular}

Data are presented as mean (SD) or median [interquartile range]

over 0 to $6 \mathrm{~h}$ after procedure commencement, the primary end point, did not vary significantly between the active and placebo groups [mean (standard deviation): 3.2 (2.5) vs. $3.1(2.2), p=0.89]$. Similarly, PI at $24 \mathrm{~h}$ did not show a significant difference (median [interquartile range (IQR)]: 1.1 [0.8-2.7] and 1.5 [0.2-4.7], $p=0.98$ ) (Table 2).

The oxycodone group experienced significantly more nausea than the placebo group (median [IQR]: 0.35 [0-1.95] vs. 0 [0-0.1], respectively, $p=0.035$ ). A significantly greater proportion of participants from the oxycodone group experienced vomiting (30\%) compared with counterparts receiving placebo $(5 \%) ;(p=0.044)$ (Table 3$)$. 
Table 3 Opioid requirement, opioid related side effects and suitability for discharge

\begin{tabular}{|c|c|c|c|}
\hline Characteristic & $\begin{array}{l}\text { Preoperative } \\
\text { placebo }\end{array}$ & $\begin{array}{l}\text { Preoperative } \\
\text { opioid }\end{array}$ & $p$ \\
\hline $\begin{array}{l}\text { Total opioid } \\
\text { requirement (oral } \\
\text { morphine equivalents) }\end{array}$ & $22.5[15-46.5]$ & $64.5[45-90]$ & $<0.0001$ \\
\hline Nausea $0-24 \mathrm{~h}$ & $0[0-0.1]$ & $0.35[0-1.95]$ & 0.035 \\
\hline Vomiting $0-24 \mathrm{~h}$ & $5 \%$ & $30 \%$ & 0.044 \\
\hline Pruritus & $0 \%$ & $5 \%$ & 1.00 \\
\hline Postprocedure sedation & $0[0-1]$ & $1[0.5-1]$ & 0.89 \\
\hline $\begin{array}{l}\text { Suitability for discharge } \\
\text { at } 24 \mathrm{~h}\end{array}$ & $35 \%$ & $11 \%$ & 0.07 \\
\hline
\end{tabular}

Data are presented as mean (SD), median [interquartile range] or proportion as appropriate

Pruritus was of low incidence in both groups, with the difference being nonsignificant $(5 \%$ in the oxycodone group vs. $0 \%$ in the placebo group; $p=1.00$ ), while sedation levels did not vary significantly between the two groups, with a median (IQR) score of $0[0-1]$ in the placebo group compared to $1[0.5-1](p=0.89)$ (Table 3). No patients experienced respiratory depression $(p=1.00)$. Overall opioid requirement over $24 \mathrm{~h}$, measured as oral morphine equivalent, was significantly greater in the oxycodone group (median [IQR]: 64.5 [45-90] mg vs. 22.5 [15-46.5] mg, $p<0.0001)$.

Although the proportion of participants eligible for discharge after $24 \mathrm{~h}$ was less in the group receiving oxycodone (11 vs. $35 \%)$, this difference was not statistically significant $(p=0.07)$ and no participants required more than a single night's stay in the hospital (Table 3).

Mean satisfaction with pain management did not vary significantly between the oxycodone and placebo groups (mean (SD): 8.3 (1.2) and $7.8(2.6), p=0.43$ ). Similarly, level of satisfaction with the procedure, sedation and analgesia did not vary significantly between groups (median [IQR]: 3.5 [3-4] and 4 [3-4], $p=0.65$ ).

There were two adverse events. One patient experienced persistent pain, nausea and vomiting and presented to the emergency department $2 \mathrm{~h}$ after discharge. The patient was treated with analgesics and antinausea medication, was discharged and reported a full recovery when contacted the next day. The other patient was involved with the procedure that was technically unsuccessful.

\section{Discussion}

Preemptive oral oxycodone was found not to reduce PI during and immediately after UAE. Previous studies [1416] showed that morphine PCA provided superior PI ratings after UAE in a nonrandomized setting compared with fentanyl PCA [16]. In the remaining randomized controlled studies, morphine PCA was associated with greater early PI compared with target-controlled remifentanil [15] and when compared with morphine PCA combined with localized intra-arterial lidocaine injection [19]. Morphine PCA alone showed no difference in PI compared to a combination of morphine and ketamine PCA [14]. Our approach of combining preemptive opioid analgesia with morphine PCA did not offer any advantage over morphine PCA alone; it produced greater opioid requirement and increased incidence of vomiting among participants.

Preemptive analgesia has been hypothesized as providing an advantage because pain pathways are inhibited before the onset of a painful stimulus [23]. However, there is no current evidence supporting any advantage for preemptive analgesia with opioids after two detailed reviews across a broad range of surgical procedures [23, 24]. Our findings of a lack of preemptive analgesia with oral opioid for UAE support the findings of these reviews.

The mechanisms of uterine pain with UAE are thought to involve pelvic cramping associated with postinfarction myometrial ischemia [25], a type of visceral pain. The natural history of PI associated with UAE is that it remains severe during and for several hours after UAE and then decreases in intensity [4]. This has been reflected in other studies [15] that have measured the pattern of opioid use in the $24 \mathrm{~h}$ after UAE, and also in our study where mean PCA opioid across both groups was much less in the 6 to $24 \mathrm{~h}$ period $(6.28 \mathrm{mg})$ compared to the 0 to $6 \mathrm{~h}$ period $(14.9 \mathrm{mg})$. We also provided an oral dose of oxycodone which should have provided a peak concentration at approximately $3.2 \mathrm{~h}[21,22]$, theoretically matching the time period of peak analgesic requirement. However, this did not appear to offer any analgesic advantage.

The oxycodone group in our study showed an increase in opioid requirement and was subject to significantly greater levels of nausea and vomiting, despite all subjects receiving a preprocedural dose of oral ondansetron. A study comparing morphine and fentanyl PCA for UAE described a high incidence of nausea and vomiting affecting both the morphine and fentanyl PCA groups [16]. In a postoperative survey, vomiting was ranked as the first and nausea the fourth most undesirable outcome, in contrast to postoperative pain, which was ranked third [26]. Female gender and increasing postoperative opioid requirement are the two greatest major risk factors for postoperative nausea and vomiting [27]. This suggests that there may be too much reliance on opioid techniques for analgesia for a procedure performed in a population at high risk for opioid-induced nausea and vomiting.

Epidural analgesia appears to have the greatest advantage as a preemptive analgesic because it provides reduced PI, reduces the need for supplemental analgesia and 
prolongs the time to rescue analgesia [24]. Epidural analgesia has been researched in the context of UAE with promising results but only in case series $[17,18]$ and needs to be evaluated in the context of a prospective randomized blinded study compared to morphine PCA, which is currently the most researched therapy and has been shown to be a useful control.

Nausea and vomiting are known to delay discharge from postanesthesia care units [28] and from the hospital after surgery [29]. A greater number of patients in the oxycodone group became eligible for discharge after $24 \mathrm{~h}$ in our study, but the result did not reach significance and none required more than a $24 \mathrm{~h}$ stay. Delay in discharge assumes great importance in modern practice because of associated patient morbidity and because of costs and inefficiencies associated with prolonged admission; many centers are beginning to mandate single-day admission for UAE procedures $[30,31]$.

We measured satisfaction with analgesia and with the procedure. Satisfaction with the analgesia was satisfactory. We provided information before the procedure and regular review of pain, both known to improve satisfaction with analgesia [32]. Access to PCA analgesia can improve autonomy and produce higher levels of patient satisfaction with pain management [33]. Presence of low PI, observed in both our oxycodone and placebo groups, have been previously shown to improve patient satisfaction with pain management $[34,35]$.

Satisfaction with sedation and procedure did not vary significantly between the groups and was satisfactory. There are no known comparators for satisfaction with radiological procedures. However, our patients were exposed to sedation, a form of anesthesia, and satisfaction after anesthesia has been linked to the presence of postanesthetic complications as well as severity of pain and nausea [36]. Our interpretation is that patients reported reasonable levels of satisfaction with their radiological procedure because of low complication rates.

In conclusion, we could not show any analgesic advantage for adding oral oxycodone before UAE and we have highlighted the adverse effects of nausea and vomiting with the possible implications of delayed discharge. Future studies should explore analgesic therapies that are opioid sparing or avoid opioid altogether.

Acknowledgments We acknowledge and thank Dr. Adam Sutton, Dr. Yeganeh Khavar, and Dr. Thomas M. Snow for their assistance with the collection of data.

Conflict of interest Alex H. Konstantatos, Helen Kavnoudias, James R. Stegeman, Dana Boyd, Maryann Street, Michael Bailey, Stuart M. Lyon, and Kenneth R. Thomson declare that they have no conflict of interest.

\section{References}

1. Bradley EA, Reidy JF, Forman RG et al (1998) Transcatheter uterine artery embolisation to treat large uterine fibroids. Br J Obstet Gynaecol 105:235-240

2. Bratby MJ, Belli AM (2008) Radiological treatment of symptomatic uterine fibroids. Best Pract Res Clin Obstet Gynaecol 22:717-734

3. Hald K, Langebrekke A, Klow NE et al (2004) Laparoscopic occlusion of uterine vessels for the treatment of symptomatic fibroids: initial experience and comparison to uterine artery embolization. Am J Obstet Gynecol 190:37-43

4. Worthington-Kirsch RL, Koller NE (2002) Time course of pain after uterine artery embolization for fibroid disease. Medscape Womens Health 7:4

5. Roth AR, Spies JB, Walsh SM et al (2000) Pain after uterine artery embolization for leiomyomata: can its severity be predicted and does severity predict outcome? J Vasc Interv Radiol 11:1047-1052

6. Stall L, Lee J, McCullough M et al (2011) Effectiveness of elective unilateral uterine artery embolization: a case-control study. J Vasc Interv Radiol 22:716-722

7. Ryu RK (2005) Uterine artery embolization: current implications of embolic agent choice. J Vasc Interv Radiol 16:1419-1422

8. Abramowitz SD, Israel GM, McCarthy SM et al (2009) Comparison of four embolic materials at uterine artery embolization by using postprocedural MR imaging enhancement. Radiology 250:482-487

9. Hovsepian DM, Mandava A, Pilgram TK et al (2006) Comparison of adjunctive use of rofecoxib versus ibuprofen in the management of postoperative pain after uterine artery embolization. J Vasc Interv Radiol 17:665-670

10. Verret V, Bevilacqua C, Schwartz-Cornil I et al (2011) IL6 and TNF expression in vessels and surrounding tissues after embolization with ibuprofen-loaded beads confirms diffusion of ibuprofen. Eur J Pharm Sci 42:489-495

11. Namur J, Wassef M, Pelage JP et al (2009) Infrared microspectroscopy analysis of ibuprofen release from drug eluting beads in uterine tissue. J Control Release 135:198-202

12. Borovac T, Pelage JP, Kasselouri A et al (2006) Release of ibuprofen from beads for embolization: in vitro and in vivo studies. J Control Release 115:266-274

13. Pisco JM, Bilhim T, Duarte M et al (2008) Pelvic pain after uterine artery embolization: a prospective randomized study of polyvinyl alcohol particles mixed with ketoprofen versus bland polyvinyl alcohol particles. J Vasc Interv Radiol 19:1537-1542

14. Jensen LL, Handberg G, Helbo-Hansen HS et al (2008) No morphine sparing effect of ketamine added to morphine for patient-controlled intravenous analgesia after uterine artery embolization. Acta Anaesthesiol Scand 52:479-486

15. Lipszyc M, Winters E, Engelman E et al (2011) Remifentanil patient-controlled analgesia effect-site target-controlled infusion compared with morphine patient-controlled analgesia for treatment of acute pain after uterine artery embolization. Br J Anaesth 106:724-731

16. Kim HS, Czuczman GJ, Nicholson WK et al (2008) Pain levels within 24 hours after UFE: a comparison of morphine and fentanyl patient-controlled analgesia. Cardiovasc Interv Radiol 31:1100-1107

17. Nagao T, Ohwada T, Kitazono M et al (2005) Thoracic epidural analgesia is effective in perioperative pain relief for uterine artery embolization [in Japanese]. Jpn J Anesthesiol 54:156-159

18. Saito S, Chiba A, Hayakawa S et al (2004) Pain control with epidural anaesthesia for uterine artery embolization [in Japanese]. Jpn J Anesthesiol 53:391-395 
19. Keyoung JA, Levy EB, Roth AR et al (2001) Intraarterial lidocaine for pain control after uterine artery embolization for leiomyomata. J Vasc Interv Radiol 12:1065-1069

20. Staahl C, Christrup LL, Andersen SD et al (2006) A comparative study of oxycodone and morphine in a multimodal tissue-differentiated experimental pain model. Pain 123:28-36

21. Bleinheim Pharmacal Inc (2010) Oxycodone hydrochloride. Pharmacokinetics and metabolism. In: Oxycodone Product information. Bleinheim Pharmacal Inc. Revised Jan 2010

22. Pantawala AE, Duby J, Waters D, Erstad BL (2007) Opioid conversions in acute care. Ann Pharmacother 41:255-266

23. Moiniche S, Kehlet H, Berg Dahl J (2002) A qualitative and quantitative systematic review of preemptive analgesia for postoperative pain relief. Anesthesiology 96:725-741

24. Ong CKS, Lirk P, Seymour RA, Jenkins BJ (2006) The efficacy of preemptive analgesia for acute postoperative pain management: a meta-analysis. Anesth Analg 100:757-773

25. Ruuskanen A, Sipola P, Hippeläinen M et al (2009) Pain after uterine fibroid embolisation is associated with the severity of myometrial ischaemia on magnetic resonance imaging. Eur Radiol 19:2977-2985

26. Macario A, Weinger M, Carney S, Kim A (1999) Which clinical anesthesia outcomes are important to avoid? The perspective of patients. Anesth Analg 89:652-658

27. Roberts GW, Bekker TB, Carlsen HH et al (2005) Postoperative nausea and vomiting are strongly influenced by postoperative opioid use in a dose related manner. Anesth Analg 101: 1343-1348
28. Gan TJ, Meyer T, Apfel CC et al (2003) Consensus guidelines for managing postoperative nausea and vomiting. Anesth Analg 97:62-71

29. Shaikh S, Chung F, Imarengiaye $C$ et al (2003) Pain, nausea, vomiting and ocular complications delay discharge following ambulatory microdiscectomy. Can J Anesth 50:514-518

30. Pron G, Mocarski E, Bennett J, Ontario UFE Collaborative Group et al (2003) Tolerance, hospital stay, and recovery after uterine artery embolization for fibroids: the Ontario Uterine Fibroid Embolization Trial. J Vasc Interv Radiol 14:1243-1250

31. Klein A, Schwartz ML (2001) Uterine artery embolization for the treatment of uterine fibroids: an outpatient procedure. Am J Obstet Gynecol 184:1556-1560

32. Sjoling M, Nordahl G, Olofsson N, Asplund K (2003) The impact of preoperative information on state anxiety, postoperative pain and satisfaction with pain management. Patient Educ Couns 51:169-176

33. Chang AM, Ip WY, Cheung TH (2004) Patient-controlled versus conventional intramuscular injection: a cost effectiveness analysis. J Adv Nurs 46:531-541

34. Thomas T, Robinson C, Champion D et al (1998) Prediction and assessment of the severity of post-operative pain and of satisfaction with pain management. Pain 75:177-185

35. Jamieson RN, Mitchell R, Hoopman P et al (1997) Assessment of postoperative pain management: patient satisfaction and perceived helpfulness. Clin J Pain 13:229-236

36. Myles PS, Williams DL, Hendrata M et al (2000) Patient satisfaction after anaesthesia and surgery: results of a prospective survey of 10811 patients. Br J Anaesth 84:6-10 